\title{
Anomalous absorption in ECRH experiments due to parametric excitation of localized $U H$ waves
}

\author{
E. Gusakov, A. Popov \\ Ioffe Institute, St. Petersburg, Russia, evgeniy.gusakov@mail.ioffe.ru
}

Electron cyclotron resonance heating (ECRH) is a technique being widely utilized nowadays in magnetic fusion experiments. It is also considered for application in ITER, for plasma heating and neoclassical tearing mode control. The analysis [1-3] of different parametric decay instabilities (PDIs) potentially dangerous for mm-waves at the ECR frequencies in the presence of the monotonous plasma profiles revealed the typical microwave power threshold in the range much higher than $1 \mathrm{MW}$. This is due to huge energy loss of the interacting waves from the decay region. The typical heating system output power range available up to now, even if the gyrotrons are joined into a group, is substantially lower than the predicted PDI power threshold value. Thus, it has been taken as known until very recently that the ordinary or extraordinary electromagnetic wave propagation and absorption in the absence of the UHR being well described by linear theory is predictable in detail.

However, during the last decade many observations have demonstrated the anomalous phenomena during ECRH. Firstly, the fast ion generation and ion heating were observed at the TJ-II stellarator [4] and the TCV tokamak [5] during the $2^{\text {nd }}$ harmonic Xmode ECRH pulse under conditions when energy exchange between electrons and ions should be very low. Secondly, the observations of the backscattering signal down - shifted from the pump frequency by approximately $1 \mathrm{GHz}$ and correlated to the MHD mode rotation were reported $[6,7]$ in the $200-600$ $\mathrm{kW}$ level $2^{\text {nd }}$ harmonic X-mode ECRH experiment in the TEXTOR tokamak and interpreted in terms of anomalous backscattering of the pump EC wave. The anomalous scattering of the X-mode in the ASDEXUpgrade tokamak $2^{\text {nd }}$ harmonic ECRH experiment has been recently reported as well $[8,9]$.

The attempts of theoretical explanation of these anomalous phenomena have been undertaken during recent years [10-13]. Several theoretical models demonstrating the possibility of excitation of the lowthreshold PDIs which lead to generation of the electron and ion Bernstein waves had been proposed. The cornerstone of them is taking full account of a nonmonotonous plasma density profile often observed in the ECRH experiments and originated due to different physical mechanisms, among which are features of plasma confinement in the magnetic island [14] or the electron pump-out effect manifesting itself as a consequence of the anomalous convective particle flux from the ECR layer at the intensive ECRH. The poloidal magnetic field inhomogeneity is a key element of the theory as well. Based on these two elements it was shown that two- or even three-dimensional (2D or 3D) trapping of either a daughter ion Bernstein wave (IBW) or a daughter electron Bernstein wave (EBW) due to a specific multidimensional plasma inhomogeneity can be responsible for excitation of several convective and absolute PDIs possessing thresholds drastically less than those predicted by the standard model.

These theoretical results expanded significantly the standard theory which ignored peculiarities of a density profile and geometry of toroidal devices. Though the developed theoretical models demonstrated the principal possibility of the low-threshold PDI excitation in the $2^{\text {nd }}$ harmonic $\mathrm{X}$ mode ECRH experiments at toroidal devices, they couldn't describe the main features and details of the observed anomalous effects. Predicted fast parametric instabilities were shown to be convective. The most dangerous absolute PDIs were demonstrated to have the growth rates comparable with the inverse characteristic times of the MHD and drift processes.

The relative crisis in development of the theory was, apparently, overcome in $[15,16]$ pointing out to the possibility that the backscattering signal generation as well as the anomalous absorption in ion channel could be a consequence of secondary nonlinear process, that accompanies a primary low - threshold absolute PDI of a different nature. A hint to this primary instability is provided experimentally in [7] demonstrating the most intensive backscattering at the plasma density in the magnetic island slightly exceeding the UHR value for half the pump frequency. In $[15,16]$ it was realized that at these conditions the low-threshold pump wave decay into the two upper hybrid (UH) waves is possible.

In the present paper we review recent results of the two-plasmon PDI theory development [15-24]. We introduce a model taking into account, as distinct from the standard theory [1-3], the presence of a nonmonotonous density profile, which always exist on the discharge axis or may be present due to the magnetic island or the density pump-out effect. We interpret the generation of backscattering signal and the anomalous ion heating, as a result of secondary nonlinear processes that accompany a primary lowthreshold two-UH-plasmon PDI driven by the pump $\mathrm{X}$-mode. The threshold of the primary PDI is shown to be smaller than the one predicted in [1] due to the UH wave trapping in radial direction $[15,16]$. It is also shown that in the presence of the finite-size pump beam the most dangerous absolute instability could be excited. The threshold of this instability is determined in hot fusion plasma not by the UH wave damping, but by diffractive losses along the magnetic field and could be as small as several tens $\mathrm{kW}$. The twoplasmon decay growth rate is very high, in the range 
of $10^{8} \mathrm{~s}^{-1}$. The primary PDI growth enhancing the UH wave fluctuations from the thermal noise level is saturated in our model due to the cascade of secondary decays of the daughter UH wave that leads to excitation of the secondary UH and ion Bernstein (IB) waves [17-20]. The threshold of this spontaneous parametric frequency down-conversion can be easily overcome for the secondary UH waves, if their radial trapping is possible. The number of the consecutive cascades is limited by the violation of the trapping condition. A set of equations describing the cascade is derived and solved numerically. The results of numerical modeling are shown to be in agreement with analytical estimations of the growth rates of the initial and secondary parametric decays and with the saturation level $[19,20]$. The coupling of different daughter $\mathrm{UH}$ waves is responsible in the model for generation of the backscattered $\mathrm{X}$ wave. This mechanism appears capable of reproducing the fine details of the frequency spectrum of the anomalously reflected $\mathrm{X}$ wave and of the absolute value of the backscattering signal observed at TEXTOR [18]. It also predicts substantial (up to $25 \%$ ) anomalous pump absorption due to the primary and secondary UH wave generation and their absorption by electrons in the vicinity of the density local maximum. The obtained anomalous absorption efficiency is large enough to consider seriously the pump wave depletion, as additional mechanism of the PDI saturation. The performed calculation results in reduction of the predicted anomalous absorption from $25 \%$ to $22 \%$ at the microwave power launched into the plasma in TEXTOR experiment [21].

It is also shown that the low-threshold absolute two-plasmon decay parametric instabilities could be excited not only in magnetic islands, but in plasma blobs or filaments elongated in the direction of magnetic field. This effect is investigated in the model experiment at the linear plasma device "Granite", where the low threshold excitation of the twoplasmon PDI leading to anomalous absorption higher than $90 \%$ is demonstrated [22].

Based on the developed approach we analyze a more general case when only one of the UH waves is trapped in the vicinity of the density maximum whereas the second daughter wave is able to leave this region. It is shown that under these conditions excitation of the absolute two-plasmon PDI is also possible $[23,24]$ and can lead to substantial anomalous absorption.

Based on the proposed model one could expect a high level of excitation of UH waves at the frequency close to the half pump wave frequency and therefore substantial emission of electromagnetic waves at this frequency due to the linear wave conversion in the UH resonance. The nonlinear coupling of the daughter UH waves with the pump could lead to the measurable level of the plasma emission at the $3 / 2$ harmonic of the pump, as it happens in the laser driven inertial fusion experiments [25]. The secondary IB waves excited in the cascade mechanism of the two-plasmon PDI saturation directly transfer the pump power to the ion component which can explain the anomalous fast ion generation at TCV and TJ-II.

It should be noted that the trapped UH waves could be excited in the experiments on the O-mode ECRH at the fundamental harmonic as well. The threshold in this case is higher (several hundred $\mathrm{kW}$ depending on the plasma parameters) than for the Xmode pump whereas the growth rate is large enough (in the range of $10^{7} \mathrm{~s}^{-1}$ ) to expect the non-linear saturation of the instability [26].

The authors would like to acknowledge the financial support of the Russian Science Foundation project 16-12-10043 and of the Ioffe Institute.

\section{References}

1. M. Porkolab, B.I. Cohen Nucl. Fusion 198828239

2. Cohen B I, Cohen R H, Nevins W M and Rognlien T D 1991 Rev. Mod. Phys. 63949

3. Litvak A G, Sergeev A M, Suvorov E V, Tokman M D and Khazanov I V 1993 Phys. Fluids B 54347

4. D. Rapisarda et al., Plasma Phys. Control. Fusion 2007 49, 309

5. A. N. Karpushov et al. Proc. of $33^{\text {rd }}$ EPS Conference on Plasma Physics, 2006 30I P-1.152

6. E. Westerhof, S. Nielsen, J. W. Oosterbeek et al. Phys. Rev. Lett. 2009103125001

7. S.K. Nielsen, M. Salewski, E. Westerhof et al. Plasma Phys. Control.Fusion 201355115003

8. V. Furtula et al., The Review of scientific instruments $\mathbf{8 3}$, 013507 (2012);

9. S.K. Nielsen et al. 2014 In Proc. of 9th Workshop "Strong Microwaves and Terahertz Waves: Sources and Applications" July 24-30 2014 Nizhny Novgorod

10. E.Z. Gusakov and A.Yu. Popov, Phys. Rev. Lett. 105, 115003 (2010);

11. E.Z. Gusakov and A.Yu. Popov, Nucl. Fusion 51, 073028 (2011);

12. E. Gusakov, A. Popov, EPL 99, 15001 (2012);

13. E.Z. Gusakov, A.Yu. Popov, A.N. Saveliev, Plasma Phys. Control. Fusion 56, 015010 (2014)]

14. M Yu Kantor 2009 Plasma Phys. Control. Fusion 51 055002

15. A.Yu. Popov, E Z Gusakov J. Exp. Theor. Phys. 120 (2015) 147

16. A.Yu. Popov, E.Z. Gusakov 2015 Plasma Phys. Control. Fusion 57025022

17. A.Yu. Popov, E Z Gusakov J. Exp. Theor. Phys. 121, (2015), 362

18. E Z Gusakov and A Yu Popov Physics of Plasmas 23, (2016) 082503

19. E Z Gusakov and A Yu Popov Plasma Phys. Control. Fusion 59 (2017) 025005

20. E. Z. Gusakov, A. Yu. Popov, M. A. Irzak JETP, 123, (2016) 723

21. E Z Gusakov and A Yu Popov submitted to Plasma Phys. Control. Fusion

22. L.V. Simonchik et al. 2017 In Proc. of 10th Workshop "Strong Microwaves and Terahertz Waves: Sources and Applications" July 17-22 2017 Nizhny Novgorod

23. A Yu Popov and E Z Gusakov EPL, 116 (2016) 45002

24. A. Yu. Popov and E. Z. Gusakov JETP Letters, 105, (2017) 78

25. E Z Gusakov Sov. Tech. Phys. Lett. 3 (1977) 504

26. E Z Gusakov, A Yu Popov, A N Saveliev and E V Sysoeva Plasma Phys. Control. Fusion, 59, (2017) 075002 